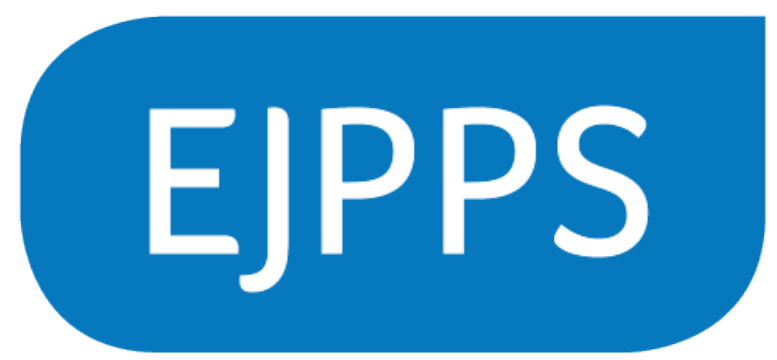

EUROPEAN JOURNAL OF

PARENTERAL AND

I PHARMACEUTICAL SCIENCES

EJPPS - European Journal of Parenteral and Pharmaceutical Sciences Volume 26 Issue 3

https://www.ejpps.online/post/vol26-3-a-comparison-of-the-bacterial-contamination-ofthe-surface-of-cleanroom-operators-garments

https://doi.org/10.37521/ejpps.26302

\title{
A Comparison of the Bacterial Contamination of the Surface of Cleanroom Operators' Garments following Donning with and without Gloves.
}

Dr Laurie M. Smith School of Pharmacy and Life Sciences, Robert Gordon University, UK

Corresponding Author: Dr Laurie M. Smith

Lecturer in Pharmaceutical Sciences

School of Pharmacy and Life Sciences,

Sir lan Wood Building,

Robert Gordon University,

Garthdee Road,

Aberdeen,

AB10 7GJ

Tel: 07751698048

Email: $\quad$ I.smith50@rgu.ac.uk 


\section{Authors and Affiliations}

Dr Noëlle H. O' Driscoll

Lecturer in Pharmaceutical Microbiology

School of Pharmacy and Life Sciences,

Sir lan Wood Building,

Robert Gordon University,

Garthdee Road,

Aberdeen,

AB10 7GJ

Prof Andrew J. Lamb

Head of Graduate School

Graduate School,

Ishbel Gordon Building,

Robert Gordon University,

Aberdeen,

AB10 7QE.

This investigation was undertaken in the Cleanroom Facility at the School of Pharmacy and Life Sciences, Technical Building, Schoolhill, Aberdeen, AB10 1FE.

\section{Research Funding}

This research project did not receive any specific grant from funding agencies in the public, commercial, or not-for-profit sectors. However, the study was funded by the Research Committee from the School of Pharmacy and Life Sciences for which thanks are offered.

\section{Conflict of Interest Declaration}

The authors declare that they have no competing interests.

\section{Ethical Approval}

A research ethics self-assessment (RESA) form was submitted in respect to the Robert Gordon University's Research Ethics Policy. This application was reviewed and approved by the Ethical Review Panel of the School of Pharmacy and Life Sciences.

\section{Acknowledgements}

The authors would like to thank Miss Hannah Prescott, Miss Mary Tully and Dr. Tina Lowes for their technical assistance and contributions throughout this study. The authors would also like to thank Dr. Christine Alexander for her advice and expertise, as well as Dr. Neil McEwan for his constructive review of the manuscript prior to submission. 


\section{Abstract}

\section{Background}

Specialist cleanroom garments are a potential vector for transmission of microorganisms within these facilities. In order to maintain the low bioburden of such clothing it has been perceived best practice for operators to dress wearing sterile cleanroom gloves. However, the efficacy of such glove use upon the resultant bacterial contamination of the surface of cleanroom garments has not previously been evaluated.

\section{Aim}

To compare surface bacterial contamination of cleanroom garments following their donning by operators dressing with or without gloves.

\section{Methods}

Following prior handwashing and systematic donning of cleanroom clothing by operators dressing wearing either no gloves, non-sterile nitrile gloves or sterile cleanroom latex gloves, a direct agar contact method was immediately undertaken to test garment surfaces at 7 specific sites. Following incubation bacterial levels were suitably quantified.

\section{Findings}

Comparing levels of growth displayed on plates used to test the surface of cleanroom garments worn by operators dressing with no gloves, non-sterile gloves or sterile cleanroom gloves, no significant difference was observed between the percentage of contact plates displaying growth and the levels of growth observed on plates, from any of the sites tested.

\section{Conclusion}

Omission of gloves in line with a systematic handwashing procedure prior to the cleanroom garment donning process, may result in modest economic and environmental gain coupled with a slightly less burdensome procedure. However, this is reliant on rigorous adherence to handwashing protocol and assessment of associated risk factors.

Keywords - Gloves, Cleanroom, Garments, Donning, Bacterial, Contamination. 


\section{Introduction}

Cleanroom operators are well established as being the predominant source of contamination within these environments ${ }^{[1-8]}$. It is reported that around $80 \%$ of cleanroom particulates are human in origin ${ }^{[9]}$, comprised of dead skin cell squames ${ }^{[1,4,5]}$ and accompanying microorganisms from the skin microbiome ${ }^{[10]}$. These particles are constantly shed by operators due to continuous epithelial cell growth and renewal ${ }^{[11]}$.

\subsection{Cleanroom Garments}

To prevent release of these contaminants the cleanroom workforce is required to wear a specific arrangement of specialist clothing ${ }^{[12]}$. The integrated clothing systems form a protective physical barrier between the operator and environment. Whilst particulate reduction efficacy of these garments is reported, their capacity to retain all operator associated particles is lacking ${ }^{[1,2,5,8,13,14]}$. For instance, operatives working in the cleanroom environment fully dressed in a suit, hood, boots, and gloves have been shown to elevate airborne particle concentration levels by over 1700 particles $/ \mathrm{m}^{3}$, omitting in the region of 17,000 particles per minute ${ }^{[2]}$.

\subsection{Bacterial Contamination of Cleanroom Garments}

The polyester fibres of reusable cleanroom garments provide a suitable substrate for microbial adherence and growth ${ }^{[15-17]}$, with skin commensal S. aureus viability maintained for up to 56 days following initial adherence ${ }^{[16]}$. Such bacteria from the skin microbiome penetrate through the cloth and contaminate the outer surface of cleanroom garments ${ }^{[6,18]}$. These clothing fibres in turn become vectors for microbial transmission ${ }^{[19]}$, compromising the sterile environment and potentially the product via airborne dissemination ${ }^{[20]}$ or direct surface-to-surface transfer ${ }^{[7,21]}$. Whyte and Eaton earlier confirmed the ability of cleanroom garments to directly transfer bacteria to hard surfaces with a 0.6 mean transfer coefficient ${ }^{[7]}$.

\subsection{Use of Gloves to Prevent Microbial Contamination}

To maintain integrity of cleanroom garments, the items are donned in a systematic manner which intends to minimise the potential for contamination of the exterior surface. Perceived best practice involves operators dressing whilst wearing sterile cleanroom gloves after rigorous methodical handwashing. The gloves selected for use are specifically manufactured to be low in extractable particulates and contaminants. Several earlier studies have compared efficacy of sterile with nonsterile glove types in prevention of spread of microorganisms via the hand-borne route ${ }^{[22-26]}$. However, these studies were undertaken with the purpose to investigate infection control between hospital-based healthcare workers and patients. Studies which have previously examined cleanroom gloves have only done so with respect to their technical properties ${ }^{[27-29]}$ rather than for their ability to reduce microbial transfer. Despite gloved hands reported as a transmission mode within the cleanroom environment ${ }^{[7,30]}$ the efficacy of glove use with respect to the contamination of cleanroom garments during their donning has not previously been investigated. Those studies which have examined the contamination of cleanroom garments sought to do so either with respect to operator gender ${ }^{[18]}$ or time spent working in the cleanroom facility ${ }^{[6]}$ with no consideration of dressing regimes.

\section{Aim and Objectives}

The purpose of this study was to compare the bacterial contamination of the surface of cleanroom operators' garments at selected sites following their donning by operators dressing 
with or without gloves. The investigation examined both sterile and non-sterile gloves as well as no glove use.

\section{Methods}

Contact plates were used to test the exterior surface of cleanroom garments donned by operators dressing wearing no gloves, non-sterile gloves or sterile cleanroom gloves. Three test subjects, all experienced in the process of gowning, dressed on nine separate occasions after washing hands using a standardised handwashing protocol incorporating antibacterial soap. For each test subject on three occasions no gloves were worn following handwashing, during the next three occasions nonsterile gloves were worn and the final three occasions involved the use of sterile cleanroom gloves prior to dressing. With 7 sites tested (Figure 3), for each of the 27 dressing events this yielded a total of 189 individual contact plate test events. Additionally, hand cleanliness following hand washing with and without glove donning and prior to and following garment donning was monitored using finger dabs on nutrient agar plates. In addition, contact plates were used to confirm the low bioburden of cleanroom garments having undergone industrial decontamination (Fishers Services Ltd, Cupar, UK).

\subsection{Contact Plate and Nutrient Agar Plate Preparation}

Contact plates were aseptically prepared by pipetting $13 \mathrm{~mL}$ of molten sterile Nutrient Agar (Oxoid Ltd, Baskingstoke, UK) into the base of a $55 \mathrm{~mm}$ contact plate (ThermoFisher Scientific, Newport, UK) to form a convex surface. Nutrient agar plates were prepared by pouring $\sim 25 \mathrm{ml}$ of molten sterile nutrient agar (Oxoid Ltd, Hampshire, UK) into the base of a $90 \mathrm{~mm}$ diameter triple vented petri dish (Fisher Scientific Ltd, Loughborough, UK). All plates were prepared and permitted to set in a laminar airflow cabinet (Labcaire Systems Ltd, Clevedon, UK). Plates were then stored at $4^{\circ} \mathrm{C}$ until two hours prior to their use when they were returned to room temperature in order to avoid condensation on the agar surface.

\subsection{Cleanroom Garments}

Cleanroom suits, boots and hoods were manufactured using ChemStat 909, the parameters and specifications of which are shown in Table 1 . This is a $100 \%$ Dacron polyester fabric employing the patented raised grid conductive fibre for static dissipation. Physical features of the suits include stud fastenings at the neck, wrist and ankles. Features of the hood and boots include tie fastenings.

Prior to use garments were thermally disinfected in barrier washers using ultrapure water $75^{\circ} \mathrm{C}$ for 6 minutes before being dried using HEPA filtered air. Garments were decontaminated and validated to Class A-ASTM F51/00 specifications and packaged individually with their integrity subsequently maintained in heat sealed, airtight, polybags (Fisher Services Ltd, Cupar, UK). 
Table 1: Fabric Parameters and Specifications of Garment Fabric (ChemStat 909).

\begin{tabular}{ll} 
Fabric Parameter & Specification \\
\hline $\begin{array}{l}\text { Weave } \\
\text { Overall Width (Inches) }\end{array}$ & Twill with antistatic grid \\
Thread Count & 60 minimum (D3774) \\
$\begin{array}{l}\text { 1" Warp } \\
\text { 1" Fill }\end{array}$ & $172 \pm 4$ \\
& $83 \pm 4$ \\
Weight oz/sq. yd & (ASTM D3775) \\
Tensile strength - Ibs & $2.95 \pm 0.15$ (ASTM D3776) \\
1" Warp & 140 minimum \\
1" Fill & 120 minimum \\
& (ASTM D5034) \\
Air Permeability @0.5" - CFM & $<15$ (ASTM D737) \\
Static Decay & 0.5 Sec \\
Surface Resistivity - Ohms per square & E9 Max (AATCC 76-1982) \\
\end{tabular}

\subsection{Determination of Bacterial Bioburden of Laundered Garments}

Contact plates were used to test the exterior surface of cleanroom suits at 6 sites and cleanroom hoods, over the oral cavity region (Figure 3). Garments were tested within a laminar airflow cabinet (Labcaire Systems Ltd, Clevedon, UK). This was disinfected with the aid of systematic unidirectional cleansing using 70\% ethanol wipes (Critical Environment Solutions Ltd, Wiltshire, UK) prior to testing. Disinfection was repeated between each garment. Prior to testing the outer packaging of each garment was disinfected using $70 \%$ ethanol wipes before the polybag was cut open at the base using sterile scissors and the garment, facing upwards, slid from its packaging into the cabinet for testing. In a systematic fashion, to avoid contamination, the garments were tested using a direct agar contact method. During testing the lid of the contact plate was removed and the surface of the agar was carefully applied to the garment surface for five seconds at constant pressure. The areas tested were subsequently wiped with a $70 \%$ ethanol impregnated wipe and the clothing sent to be laundered as per normal procedure. Contact plates were incubated at $37^{\circ} \mathrm{C}$ and examined for growth after 24 and 48 -hours.

\subsection{Standardised Handwashing and Drying Protocol}

Using elbow operated taps, operators pre-wetted hands under hot water before applying one depression of antimicrobial handwash (HiBiSCRUB ${ }^{\circ}$ antimicrobial skin cleanser, Regent Medical Ltd, Lancashire, UK) into the palm of non-dominant hand. Palms were placed together and a circular rubbing motion, maintained for approximately 10 seconds, covering the back and front of the hands and reaching 2 inches along the wrists. Placing the right-hand over the left-hand, fingers were slid back and forth 3 times between each other. The right-hand was then rotated around the left thumb 3 further times. Using the right-hand, the back of the left-hand was cleaned using a back-and-forth motion up to the wrist 3 times. This was repeated for the right-hand. Cupping both hands, the fingertips of the right-hand were placed into the cupped left-hand so that the right-hand was on top of the left-hand, with hands in opposite direction to one another. Fingertips were rubbed against the back of the opposite hand's fingers 5 times, massaging the antimicrobial soap into the nail grooves of each hand. The entirety of the hands was swept again, rubbing the inside of the inter digits 3 times. Running hot water was then applied to the wrists and allowed to run down the entirety of the hands until soap was rinsed off. Hands were then dried under hot-air hand dryer for 20 seconds. 


\subsection{Cleanroom Garment Donning}

Operators entered the changing area of the cleanroom facility wearing standardised street clothing which consisted of one thin cotton layer of loose long-sleeved t-shirt, long-legged trousers, and cotton socks. After removal of footwear, they donned over-shoes, an individually wrapped, gamma sterilised, 3-ply face mask and hair net (Critical Environment Solutions Ltd, Wiltshire UK). Operators then undertook a standardised timed handwashing and drying protocol (Section 3.3) before donning either non-sterile boxed nitrile gloves (Fisher Scientific, Loughborough, UK), KIMTECH ${ }^{\mathrm{TM}}$ pure G5 sterile latex cleanroom gloves (Basan UK, Basingstoke, UK) or omitted to wear gloves before systematically donning a reusable antistatic carbon filament polyester hood, suit, and boots.

\subsubsection{Systematic Garment Donning Procedure}

Carefully touching only the inside of the fabric with fingertips, the hood was retrieved from the packaging and donned. This was pulled over the head and secured at the back of the head using the ties. Next, the suit was retrieved from the packaging by pulling the inner fabric at the neck area with only fingertips, and once removed this was unzipped from the chest downwards and the neck/chest regions rolled outward down to the lumbus region ensuring the suit did not touch the floor. The operator then placed one leg through the leg/ankle opening and pulled the suit over the leg; this was repeated with the other leg. The operator then unrolled the suit, touching only the inside, and placed one hand through the arm/wrist opening before bringing the suit up over the arm, this was repeated with the other arm, ensuring the hood was on the inside of the suit. The suit was then zipped upwards from the lumbus region to the chest, where it was secured in place using the secure stud fastening. The stud fastenings at the wrist and ankles of the suit were then secured. One at a time, the boots were removed from the outer packaging, touching only the inner fabric of the boot, and these were then placed over the foot and suit and tied securely at the knee and ankle.

\subsection{Determination of the Bioburden of Hands Prior to and Following Cleanroom Garment Donning}

Determination of the Bioburden of Hands Prior to and Following Cleanroom Garment Donning The operator followed a standardised timed handwashing and drying protocol (Section 3.4). The operator then donned either non-sterile boxed nitrile gloves (Fisher Scientific, Loughborough, UK), $\mathrm{KIMTECH}^{\mathrm{TM}}$ pure G5 sterile latex cleanroom gloves (Basan UK, Basingstoke, UK) or omitted to wear gloves. To monitor hand contamination prior to garment donning hands were immediately tested using the finger dab testing procedure (Section 3.6.1). To monitor hand contamination levels following cleanroom garment donning the operator donned a cleanroom hood, suit and boots whilst adhering to the systematic garment donning procedure (Section 3.5.1) before testing hands using the same finger dab testing procedure (Section 3.6.1). In each case, prior to and following donning, finger dabs were repeated in triplicate for each variable; no gloves, non-sterile gloves, and sterile cleanroom gloves. Finger dab testing was also undertaking on unwashed hands prior to and following cleanroom garment donning. In the case of unwashed hands these were not washed for at least 2 hours prior to testing.

\subsubsection{Finger Dab Testing}

During finger dab testing the lid of the nutrient agar plate was removed and the tips of operators' right four fingers and thumb were pressed onto the surface of the agar for 5 seconds at constant pressure. This was repeated for the left fingers and thumb using a separate agar plate. Plates were incubated at $37^{\circ} \mathrm{C}$ and examined for growth at both 24 and 48 -hour time periods. 


\subsection{Cleanroom Garment Testing}

Immediately following their donning, each operator had the surface of the garments tested at 7 sites [1) chest, 2) umbilicus, 3) posterior cervicis, 4) lumbus, 5) left carpus, 6) right carpus and 7) oral cavity region of hood (Figure 3)] using a sterile contact plate on each occasion. During testing the lid of the contact plate was removed and the surface of the agar was carefully applied to the garment surface for five seconds at constant pressure. The areas tested were subsequently wiped with a $70 \%$ ethanol impregnated wipe (Critical Environment Solutions Ltd, Wiltshire, UK) and the clothing sent to be laundered as per normal procedure. Contact plates were incubated at $37^{\circ} \mathrm{C}$ and examined for growth at both 24 and 48 -hour time periods. At each of these time points contact plates were recorded as displaying either no (0 cfu/plate), low (1-9 cfu/plate), moderate (10-20 cfu/plate) or high-level growth (>20 cfu/plate). Results were statistically analysed using Two-Way Analysis of Variance (ANOVA) at a 95\% confidence level (GraphPad Prism 9.0 [GraphPad Software Inc., La Jolla, $\mathrm{CA}])$.

\section{Results}

\subsection{Bioburden of Laundered Cleanroom Garments}

No colonies were detected on contact plates used to test the surface of garments having undergone industrial thermal decontamination, following either 24 and $48 \mathrm{hr}$ incubation periods at $37^{\circ} \mathrm{C}$. The result confirms the low bioburden of the cleanroom garments prior to donning and hence the effectiveness of the process.

\subsection{Bioburden of Hands Prior to and Following Cleanroom Garment Donning}

As shown in Table 2 there was found to be an increase in the number of colonies observed on finger dab plates following a further 24-hour incubation period, taking the total incubation period to 48 hours. Whilst bacteria were observed on plates used to test unwashed hands, following this period, no bacteria were observed on plates used to test hands having undergone a standardised handwashing process with the omission of gloves prior to the donning process, confirming the effectiveness of the handwashing process.

Table 2: Average number of colonies on plates receiving finger dabs from unwashed hands or those having undergone a standardised handwashing technique with the omission of gloves, or donning of either non-sterile gloves or sterile cleanroom gloves both prior to and following cleanroom garment donning.

\begin{tabular}{lllll} 
& \multicolumn{2}{l}{ Average Number of CFU/Plate } & \multicolumn{2}{l}{ 48hr Incubation } \\
\cline { 2 - 5 } 24hr Incubation & Right & Left & Right & Left \\
\hline Prior to Donning & 610 & 403 & 623 & 436 \\
Unwashed Hands & 0 & 0 & 0 & 0 \\
Glove Omission & 2 & 1 & 3 & 1 \\
Non-Sterile Gloves & 0 & 0 & 0 & 0 \\
Sterile Cleanroom Gloves & 0 & & & \\
Following Donning & 121 & 127 & 154 & 159 \\
\hline Unwashed Hands & 0 & 0 & 0 & 0 \\
Glove Omission & 11 & 33 & 14 & 39 \\
Non-Sterile Gloves & 3 & 6 & 4 & 8 \\
Sterile Cleanroom Gloves & 3 & &
\end{tabular}


In the case of handwashing with the omission of gloves and the donning of cleanroom gloves, results comply with the recommended microbiological monitoring of an operating Grade A cleanroom action limit of $<1$ cfu per 5 fingers/glove ${ }^{[12]}$. The number of colonies on plates used to test hands having undergone handwashing with the subsequent donning of non-sterile gloves prior to the donning process were out with these limits. With each of the variables, prior to donning, an increased number of colonies were observed on plates used to test the non-dominant hand, whilst following donning greater numbers were recorded on plates used to test the dominant hand.

Following the donning process there was a reduction in the number of bacteria on unwashed hands, suggesting the transfer during the procedure of bacteria to other locations including the garments. No bacteria were observed on plates used to test hands having undergone handwashing with the omission of gloves following this process, in compliance with regulatory limits ${ }^{[12]}$. Out with these limits, bacteria were observed on plates used to test the hands following donning wearing sterile cleanroom gloves and non-sterile gloves, with the latter in greater abundance.

\subsection{Comparison of the Bacterial Contamination of Cleanroom Operators' Garments following Donning}

There was no significant difference observed between the number of contact plates displaying growth against the variable of no gloves, non-sterile gloves or sterile cleanroom gloves following either 24-hour and 48-hour incubation periods (Figure 1). However, a significant increase in the percentage of contact plates displaying growth at 48-hours, following a further 24-hour incubation period, was observed [** $p<0.01]$.

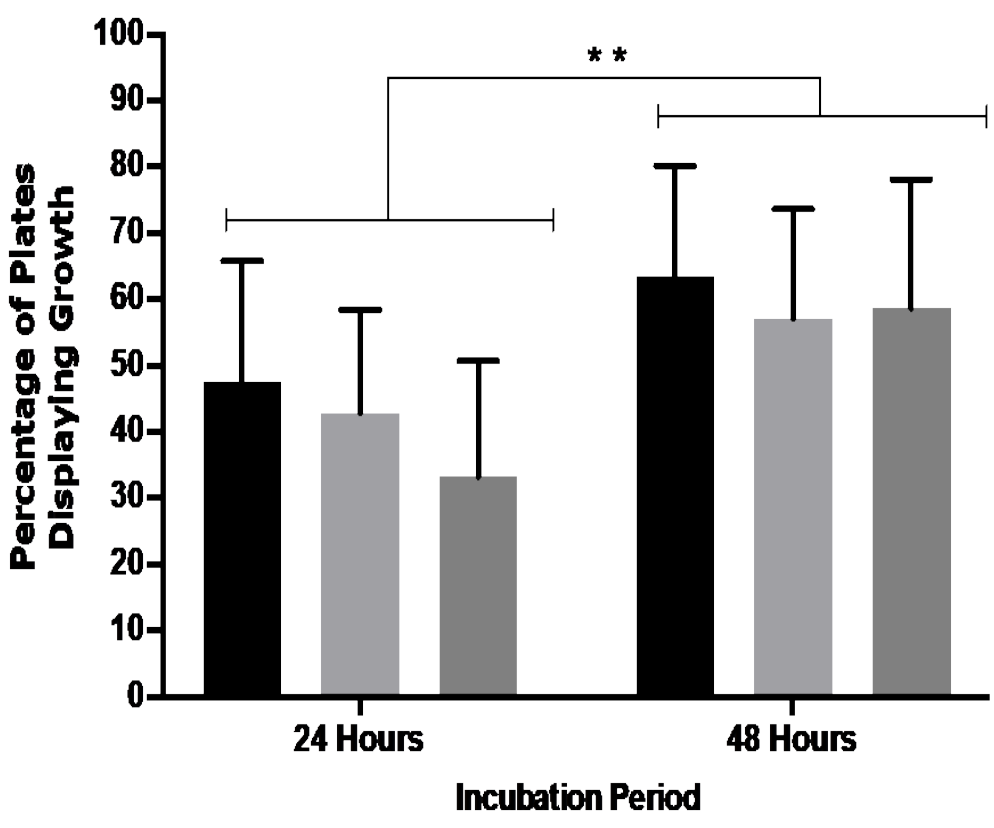

No Gloves

Non Sterile Gloves

Sterile Cleanroom Gloves

Figure 1: Comparison of the total percentage of contact plates displaying growth used to test the surface of garments donned by operators dressing wearing no gloves, non-sterile gloves or sterile cleanroom gloves following 24 -hour and 48-hour incubation periods [ ${ }^{* *} p<0.01$ ].

A comparison of the levels of growth observed on contact plates used to test the surface of the garments was also investigated following both incubation periods (Figure 2). No significant 
difference was observed between the percentage of plates displaying low (1-9 cfu/plate), moderate (10-20 cfu/plate) or high-levels of growth (>20 cfu/plate) and the variable no gloves, non-sterile gloves or sterile cleanroom gloves, following either 24-hour and 48-hour incubation periods [p>0.05].

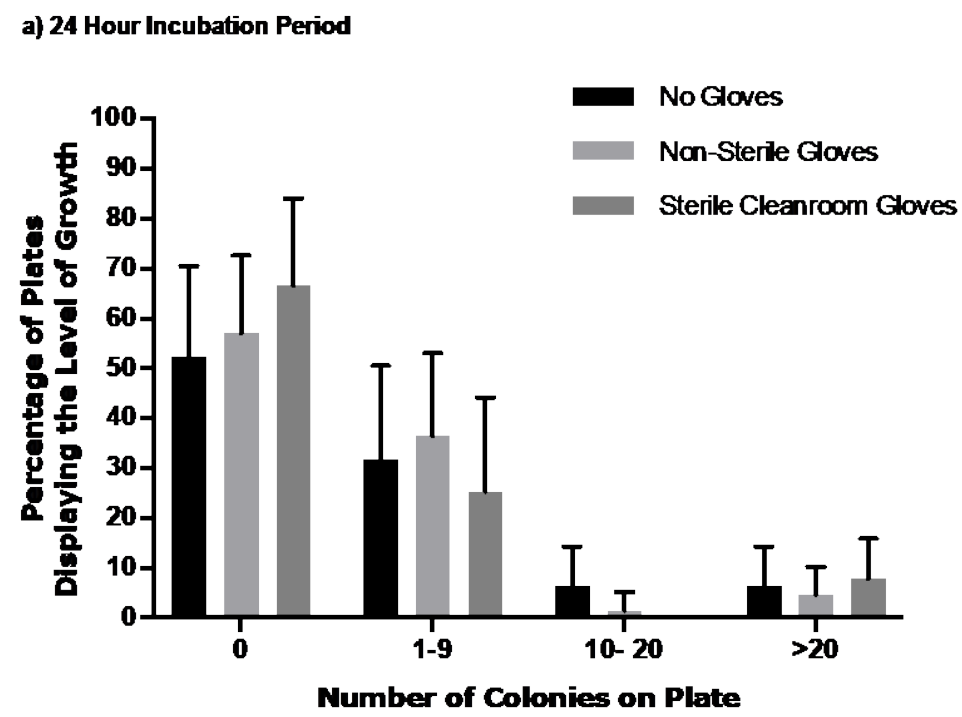

b) 48 Hour Incubation Period

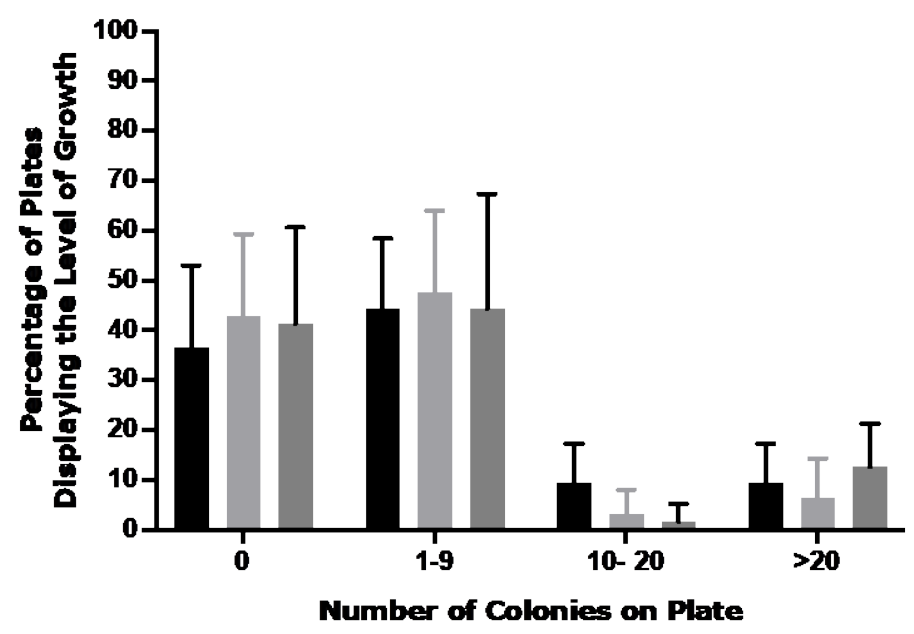

Figure 2: Comparison of the total percentage of contact plates displaying either 0 (no growth), 1-9 (low), 10-20 (moderate) or $>20$ (high-level of growth) cfu/plate used to test the surface of cleanroom garments donned by operators dressing wearing no gloves, non-sterile gloves or sterile cleanroom gloves following a) 24-hour and b) 48-hour incubation periods.

The increase in the percentage of plates displaying growth following an additional 24-hour incubation period (Figures 1 and $2 ;^{* *} p<0.01$ ) suggests use of a 24 -hour incubation period at $37^{\circ} \mathrm{C}$, as historically recommended ${ }^{[31]}$, may underestimate contamination levels on the surface of garments. For the remainder of this study only plates incubated for 48 hours were analysed. It is important to note that cleanroom environmental monitoring incubation periods temperatures, and isolation media selected may differ from this approach. However, maximum recovery efficiency was not the overall aim of this study, but rather to recover, enumerate and compare the levels of contamination 
on the surface of the garments following donning with respect to the variable with and without gloves, as was achieved.

Comparison of Bacterial Contamination of Cleanroom Garments at Specific Sites following Donning The bacterial contamination of specific garment sites with respect to glove use (and type) was also investigated (Figure 3).

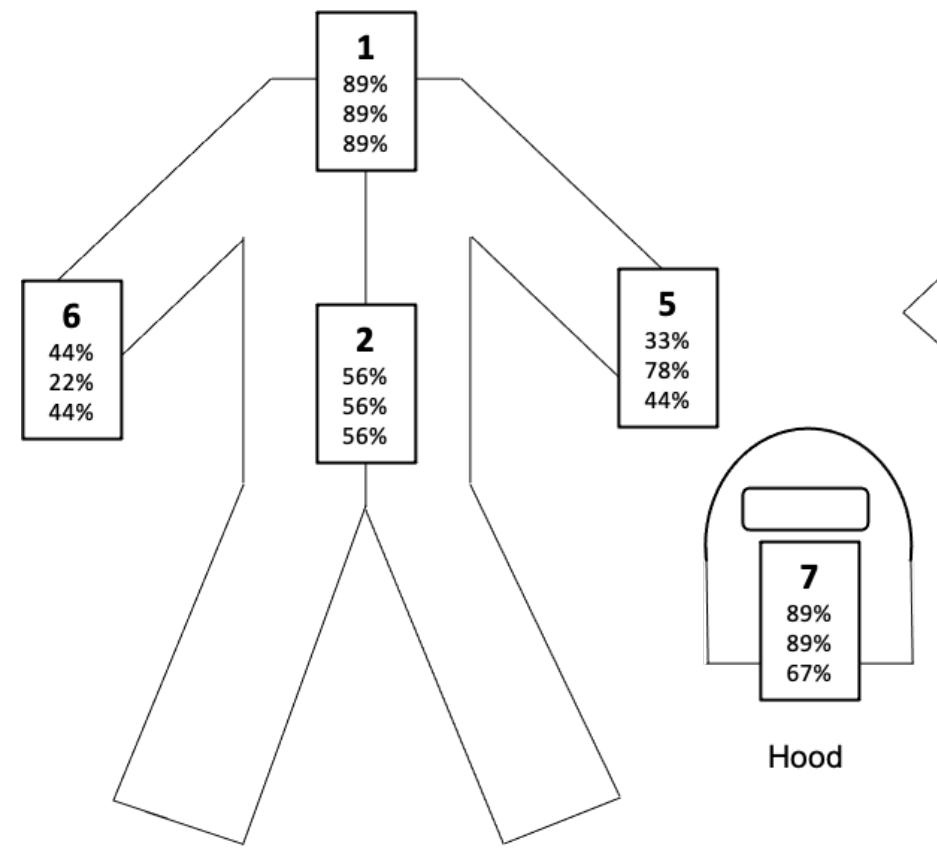

Front of Cleanroom Suit

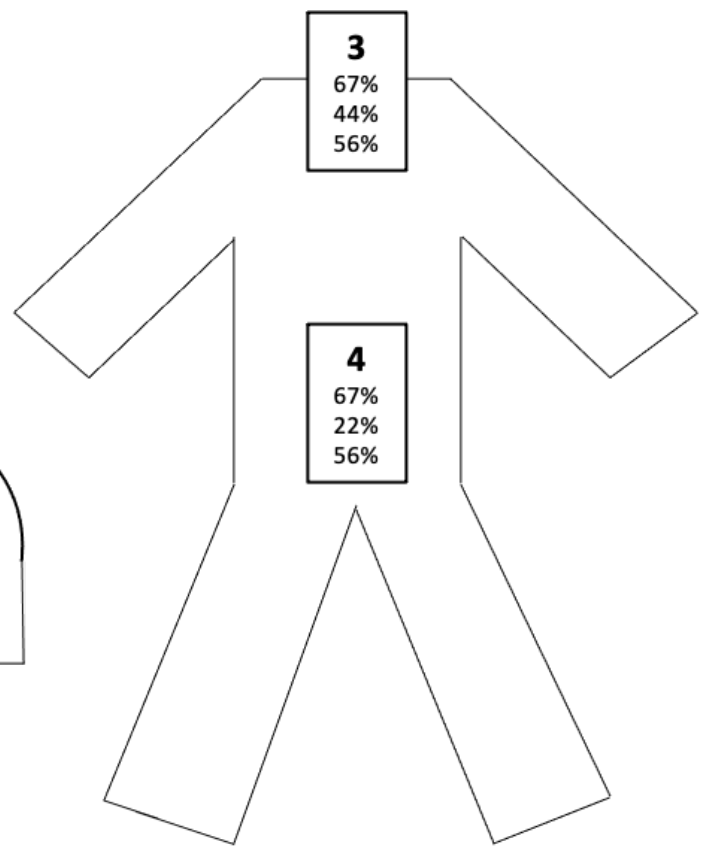

Back of Cleanroom Suit

Figure 3: Comparison of the total percentage of contact plates displaying growth at each site: 1) chest, 2) umbilicus, 3) posterior cervicis, 4) lumbus, 5) left carpus, 6) right carpus and 7) oral cavity, used to test the surface of garments donned by operators dressing wearing either no gloves (upper value), non-sterile gloves (mid value) or sterile cleanroom gloves (lower value).

No significant difference was observed between the percentage of contact plates displaying growth and the variable no gloves, non-sterile gloves or sterile cleanroom gloves, for each of the 7 garment sites tested [ $p>0.05]$. As shown in figure 3 , the highest levels of bacterial contamination in all instances were observed to be at the chest and oral cavity. To obtain further insight, a comparison of the levels of bacterial contamination at each of the specific garment sites tested was also undertaken (Figure 4). The data revealed no difference between the percentage of plates displaying low (1-9 cfu/plate), moderate (10-20 cfu/plate) or high-level growth (>20 cfu/plate) and the variable no gloves, non-sterile gloves or sterile cleanroom gloves from any of the 7 sites tested [ $p>0.05]$. 


\section{1) Chest}

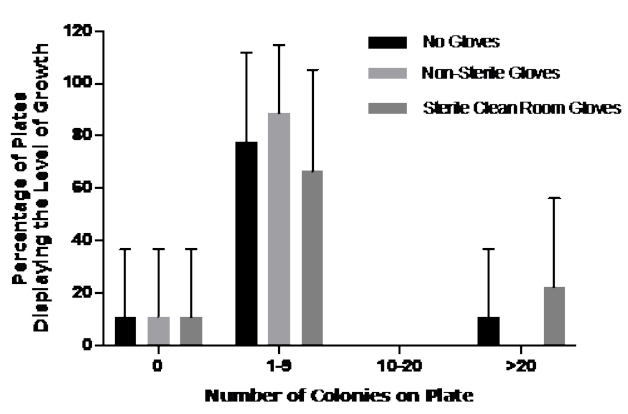

3) Posterior Cervicis

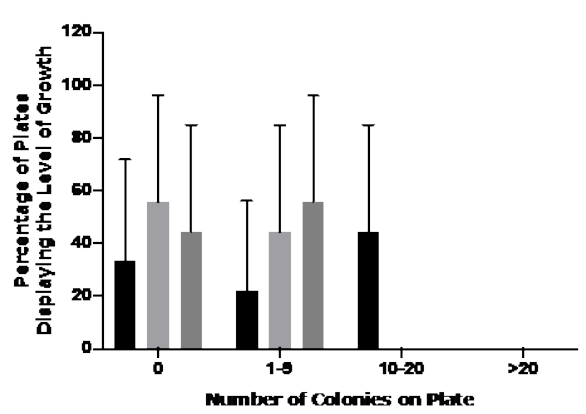

5) Left Carpus

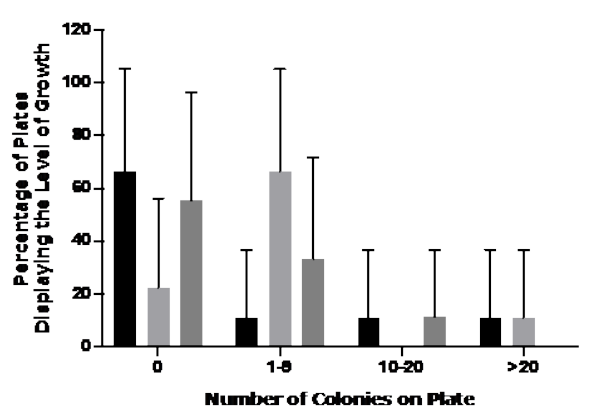

\section{2) Umbilicus}

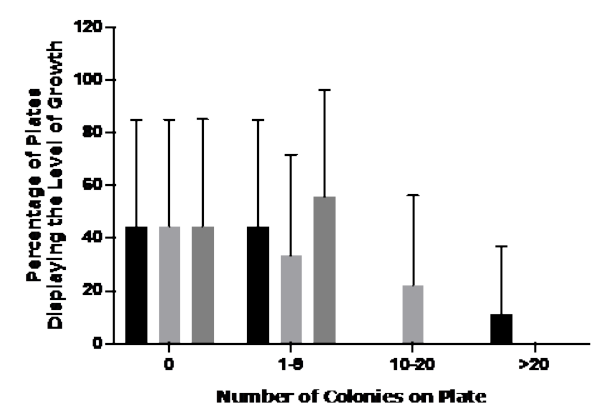

4) Lumbus

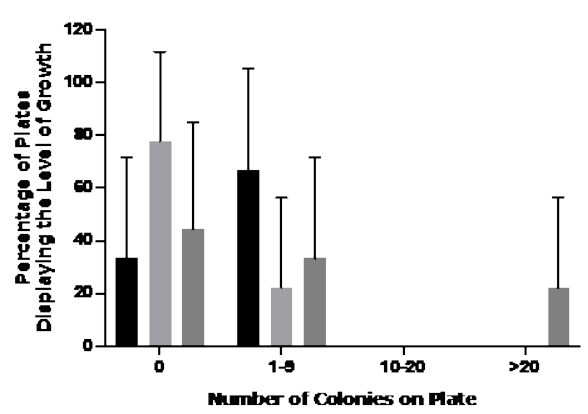

6) Right Carpus

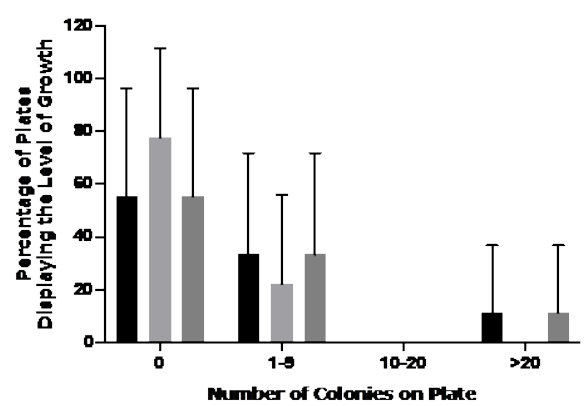

\section{7) Oral Cavity}

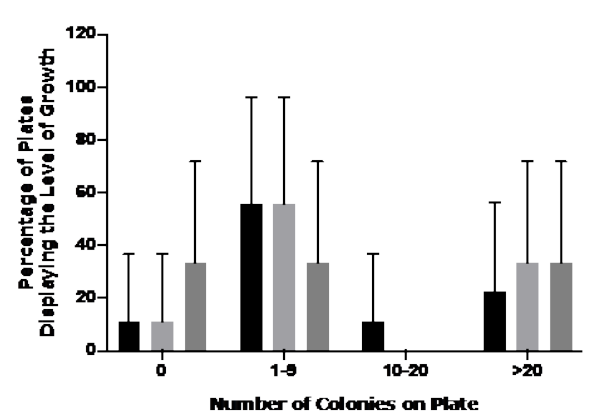

Figure 4: Comparison of the total percentage of contact plates displaying either 0 (no growth), 1-9 (low), 10-20 (moderate) or $>20$ (high-level of growth) cfu/plate used to test the 1) chest, 2) umbilicus, 3) posterior cervicis, 4) lumbus, 5) left carpus, 6) right carpus and 7) oral cavity regions of garments donned by operators dressing wearing either no gloves, non-sterile gloves or sterile cleanroom gloves. 
Of note, a higher percentage of contact plates used to test the chest and lumbus of garments donned specifically with sterile cleanroom gloves displayed high-levels of growth compared to plates used to test the same region of garments donned with non-sterile gloves or without gloves. The highest levels of growth were detected at the oral cavity region of hoods regardless of the variable no gloves, non-sterile gloves or sterile cleanroom gloves. High-levels of growth at the chest of suits and the oral cavity of hoods has previously been reported following their wear by operatives working within the cleanroom environment ${ }^{[18]}$ and therefore it is important to consider that hand borne transfer during the donning process may have contributed to this.

High-levels at the chest region of suits conceivably arise from retrieval of suits from their packaging, as well as the complex donning process ${ }^{[21]}$. Suits are retrieved from their packaging by pulling the inner fabric at the neck/chest area from the packaging with the fingertips, once removed these are unzipped from the chest downwards and the neck/chest region rolled outward down to the lumbus region were held prior to donning. Furthermore, once donned the zip is pulled back up across the chest region where it is secured using a stud fastening (being a feature of the suit type used in this study). Perhaps the higher levels detected at the lumbus region results from the suit being held specifically in this area when the operators step into the leg/ankle sections, although this requires further investigation.

As well as hand borne transfer during the donning process, high levels on hoods are also thought to arise from the abundance of facultative and aerobic species residing in the oral microbiome ${ }^{[32]}$. Levels of bacteria on the surface of cleanroom operatives' garments have been shown to increase over time ${ }^{[6]}$ as probably occurred in this case where the hood had been donned prior to suit and boots. Importantly in this respect, use of gloves or otherwise during donning would have no impact on the level of bacterial contamination that arose

\section{Discussion}

The results of the study confirm a standardised handwashing technique can decontaminate hands to a level in compliance with regulatory limits for an operational Grade A cleanroom ${ }^{[12]}$. Furthermore, no significant difference in the surface contamination of cleanroom garments and the donning variable no gloves, non-sterile gloves and sterile cleanroom gloves was observed. The findings indicate that glove use could be substituted for a rigorous standardised handwashing protocol with the omission of gloves prior to dressing, without increasing bacterial bioburden of garments. However, with adoption of such practice associated risk factors must be considered and evaluated.

\subsection{Sterile Cleanroom Glove Use as a Barrier Method to Prevent the Spread of Infection}

Despite a lack of research investigating the efficacy of sterile cleanroom gloves as a barrier method to prevent hand-borne transmission of microbial contamination, the use of sterile surgical gloves to prevent the spread of infection in general health care settings is well documented, with several previous studies reporting use of this glove type does not reduce the incidence of post clinical procedure infection rate ${ }^{[22-26,33,34]}$. However, it is difficult to draw direct comparison between glove types and practices used in health care settings and those used in pharmaceutical cleanroom facilities. In fact, this lack of clarity was the rationale for undertaking this study, as it provides some information for the context of cleanroom glove use and none the less provide others with a starting point from which to undertake more rigorous investigations.

Despite this, matters concerning physical cleanroom glove properties, such as chemical resistance ${ }^{[27]}$, tensile strength ${ }^{[28]}$ and integrity ${ }^{[28,29]}$ have previously been investigated. Of concern, physical 
failure frequencies of $30-50 \%$, relating to holes/leaks, are reported ${ }^{[28,29]}$, being threefold that observed with low-modulus nitrile gloves ${ }^{[29]}$. The reduction of plasticisers and/or oils within the rubber, reduced to lessen extractable contaminants, were suggested to be possible contributing factors ${ }^{[28,29]}$.

Furthermore, although not reported with respect to sterile cleanroom gloves specifically, problems associated with sterile glove use are well documented in the literature ${ }^{[35-39]}$ and are deemed factors worthy of attention when considering their cleanroom equivalent. In reviewing these Osman and Jenson (1999) highlight such issues ${ }^{[35]}$ including material perforations ${ }^{[36,37]}$, promoting bacterial passage over time ${ }^{[37]}$, reduction in hand and finger sensation and feel ${ }^{[39]}$, negatively affecting operator dexterity, as well as health-related concerns including rubber latex hypersensitivity ${ }^{[35]}$. Moreover, sterile glove application and removal ${ }^{[38]}$, like that of their cleanroom counterparts, can be challenging due to glove arrangement within packaging and donning without touching the rubber's exterior surface to avoid contamination.

During our study, operators observed notable differences in surface resistance between gloved and ungloved hands. This issue was reported by Carré et al. (2017) ${ }^{[40]}$, who noted increased friction between latex gloved and ungloved hands. Although investigating this between fingers and steel/glass surfaces, the dry conditions are representative of those encountered during the donning process. In fact, friction has been shown to increase the transfer of bacteria from fabric to fingertips between 2-5 times ${ }^{[21]}$ and may account for the increased bacterial numbers observed on gloved hands during our study.

\subsection{Non-Sterile Glove Use as a Barrier to Prevent the Spread of Infection}

Non-sterile glove use during donning was investigated as a slightly less expensive alternative to their more costly sterile cleanroom counterparts. However, results of the study confirm that non-sterile gloves are subject to bacterial contamination prior to wear. This is confirmed by Hughes et al. (2013), reporting recovery of skin commensal, environmental and pathogenic species from unused gloves retrieved from open boxes ${ }^{[41]}$. Additionally, problems associated with non-sterile glove use are reported ${ }^{[41-43]}$ and should be considered in their use in the cleanroom setting. High material failure rates and variability in brand performance ${ }^{[42-43]}$ are cited.

\subsection{Impact of the Ommission of Gloves on Bacterial Contamination}

The findings from the study reported here indicate that use of a standardised hand washing protocol incorporating an appropriate antimicrobial soap, with the omission of subsequent glove use, did not result in an increase in bacterial levels on the surface of cleanroom garments following donning. However, non-compliance risk factors must be considered and evaluated, with our claim relying upon the robustness and repeatability of the handwashing technique, which may not always be guaranteed.

Historically, it was Semmelweis who in the mid- $19^{\text {th }}$ century discovered the importance and effectiveness of handwashing ${ }^{[44]}$. This is now universally recognised as a simple, efficient, and costeffective manner to reduce spread of infection via the hand borne route ${ }^{[45-47]}$. However, adherence to hand washing protocol is an issue well documented in the literature, with several perceived barriers noted. However, an audit of hand hygiene practice within one UK hospital identified overuse of gloves as the primary contributing factor to the poor hand hygiene practices observed, with gloves being worn for activities where not clinically required ${ }^{[48]}$. In fact, it appears to be a common misconception that glove use increases safety and cleanliness ${ }^{[48]}$. Glove use should be in compliance with organisational policies and limited to instances where there is a requirement for wear ${ }^{[38]}$. 


\subsection{Economic and Environmental Considerations of Glove Use}

Unnecessary glove use contributes to increased expenditure to organisations and environmental waste ${ }^{[48]}$. With the average pair of non-sterile nitrile gloves normally retailing at approximately $f 0.30 / \$ 0.40{ }^{[49]}$ and sterile cleanroom gloves, considerably more expensive, at approximately $£ 1.50 / \$ 2.00$ per pair ${ }^{[50]}$, economic factors are worth consideration. Over time, as previously highlighted in the literature, the cost savings associated with the omission of gloves would be considerable ${ }^{[22,26,39,48]}$. An initiative to reduce inappropriate glove use by one UK hospital trust resulted in an annual saving of nearly $£ 100,000^{[48]}$. Costs associated with glove disposal must also be factored ${ }^{[51]}$, along with the long-term environmental impact and associated health implications ${ }^{[52-}$ 53]. If sterile cleanroom glove use during donning could be replaced by a standardised hand hygiene practice incorporating suitable antimicrobial soap, these potential benefits could be realised.

\subsection{Study Limitations}

Unfortunately, this study was limited by a number of factors. The primary limitation is linked to the relatively small number of subjects tested, necessitated by the restrictions in time to fit in with the working schedule of the clean room facility. Ideally, to ensure the validity of the findings from the small-scale study this should be repeated with a larger pool of operatives. Whilst clear statistical significance was observed with the data sets presented here, it would be wise to draw caution around overinterpretation in the absence of studies that yield similar findings.

Additionally, the number and location of garment sampling sites may have constrained the study. Unfortunately, the sites tested did not account for all areas where the gloved/ungloved hand could have touched, such as the ties on the hood and boots, and these sites should be considered in any future investigation. Whilst accepting the limitations of our study, the paper will none the less provide others with a foundation from which to undertake more rigorous investigations.

\section{Conclusion}

No significant difference in the levels of bacterial contamination of the surface of cleanroom operators' garments was observed following their donning with either no gloves, non-sterile gloves or sterile cleanroom gloves. The results indicate that omission of gloves in association with appropriate handwashing procedure, could offer modest economic and environmental gain coupled with a slightly less burdensome procedure. Adoption of this practice would be reliant on rigorous adherence to systematic handwashing and assessment of risk factors. Moreover, glove omission is not deemed standard practice in licenced facilities, perceived best practice involves sterile cleanroom glove use following rigorous methodical handwashing. 


\section{References}

1. Whyte $W$ and Hejab M. Particle and microbial airborne dispersion from people. European Journal of Pharmaceutical Sciences 2007;12(2):39-46.

2. Strauss $L$, Larkin $J$ and Zhang KM. The use of occupancy as a surrogate for particle concentrations in recirculating, zoned cleanrooms. Energy and Buildings 2011;43(11):3258-62.

3. Chen TH, Chen WP and Wang MJ. The effect of air permeability and water vapour permeability of cleanroom clothing on physiological responses and wear comfort. Journal of Occupational and Environmental Hygiene 2014;11(6):366-76.

4. Sandle T. People in cleanrooms: understanding and monitoring the personnel factor. Peer reviewed: cleanroom contamination [online]. UBM Inc, London, UK, 2014;

http://www.ivtnetwork.com/article/people-cleanrooms-understanding-and-monitoringpersonnel-factor [Accessed 16 November 2020].

5. Ramstorp M, Gustavsson M and Gudmundsson A. Particle generation from humans - a method for experimental studies in cleanroom technology. In: Indoor Air, Proceedings of the 10th International Conference on Indoor Air Quality and Climate, Beijing, 4-9 Sept, 2005. Tsinghua University Press, China, 2005;1572-1576. International Society of Indoor Air Quality and Climate. https://www.isiaq.org/docs/PDFs/1572.pdf [Accessed 17 November 2020].

6. Grangé J, Nguyen S, Bordenave J, et al. Contamination of integral protective suits in the sterile environment used for producing total parenteral nutrition bags. Le Pharmacien Hospitalier 2011;46(1):e12-18.

7. Whyte $W$ and Eaton T. Microbial transfer by surface contact in cleanrooms. European Journal of Parenteral and Pharmaceutical Sciences 2015;20(4):127-31.

8. Reinmüller B and Ljungqvist $B$. Modern cleanroom clothing systems: people as a contamination source. PDA Journal of Pharmaceutical Science and Technology 2003;57(2):114-25.

9. Bryant R. Getting the best garment service [online]. HPCi Media, London, UK, 2010; https://cleanroomtechnology.com/news/article_page/Getting_the_best_garment_service/5784 4 [Accessed 13 November 2020].

10. Whyte $W$. Settling and impaction of particles into containers in manufacturing pharmacies. PDA Journal of Pharmaceutical Science and Technology 1981;35(5):255-61.

11. Grice EA and Segre JA. The Skin Microbiome. Nature Reviews Microbiology 2011;9(4):244-53.

12. European Commission. Eudralex The Rules Governing Medicinal Products in the European Union Volume 4 EU Guidelines to Good Manufacturing Practice Medicinal Products for Human and Veterinary Use Annex 1 Manufacture of Sterile Medicinal Products (corrected version) [online]. Brussels, Belgium: European Commission 2008.

https://ec.europa.eu/health/sites/health/files/files/eudralex/vol-4/2008_11_25_gmpan1_en.pdf [Accessed 16 November 2020].

13. Ljungqvist $B$ and Reinmüller $B$. People as a contamination source: cleanroom clothing systems after 1, 25 and 50 washing / sterilisation cycles. European Journal of Pharmaceutical Sciences 2003;8(3):75-80.

14. Romano F, Milani S and Joppolo CM. Airborne particle and microbiological human emission rate investigation for cleanroom clothing combinations. Building and Environment 2020;180:106967.

15. Hsieh Y and Merry J. The adherence of Staphylococcus aureus, Staphylococcus epidermidis and Escherichia coli on cotton, polyester and their blends. Journal of Applied Bacteriology 1985;6(6):535-44.

16. Neely AN and Maley MP. Survival of enterococci and staphylococci on hospital fabrics and plastic. Journal of Clinical Microbiology 2000;38(2):724-6.

17. Schmidt - Emrich S, Stiefel P, Rupper R, et al. Rapid assay to assess bacterial adhesion on textiles. Materials 2016;9(4):249. 
18. Smith LM, O'Driscoll NH and Lamb AJ. Gender influences bacterial contamination of reusable cleanroom operators' garments following wear. European Journal of Parenteral and Pharmaceutical Sciences 2020;25(2):1-16.

19. Mitchell A, Spencer M and Edmiston Jr C. Role of healthcare apparel and other healthcare textiles in the transmission of pathogens: a review of the literature. Journal of Hospital Infection 2015;90:285-92.

20. Licina $D$ and Nazaroff WW. Clothing as a transport vector for airborne particles: chamber study. Indoor Air 2018;28(3):404-14.

21. Sattar SA, Springthorpe S, Mani S, et al. Transfer of bacteria from fabrics to hands and other fabrics: development and application of a quantitative method using Staphylococcus aureus as a model. Journal of Applied Microbiology 2001;90(6):962-70.

22. Perelman VS, Francis GJ, Rutledge T, et al. Sterile versus nonsterile gloves for repair of uncomplicated lacerations in the emergency department: a randomised controlled trial. Annals of Emergency Medicine 2004:43(3):363-70.

23. Mehta D, Chambers N, Adams B, et al. Comparison of the prevalence of surgical site infection with use of sterile versus nonsterile gloves for resection and reconstruction during mohs surgery. Dermatologic Surgery 2014;40(3):234-9.

24. Ghafouri HB, Zoofaghari SJ, Kasnavieh MH, et al. A pilot study on the repair of contaminated traumatic wounds in the emergency department using sterile versus non-sterile gloves. Hong Kong Journal Emergency Medicine 2014;21(3):148-52.

25. Heal C, Sriharan S, Buttner PG, et al. Comparing non-sterile to sterile gloves for minor surgery: a prospective randomised controlled non-inferiority trial. Medical Journal of Australia 2015;202(1):27-31.

26. Brewer JD, Gonzalez AB, Baum CL, et al. Comparison of sterile vs nonsterile gloves in cutaneous surgery and common outpatient dental procedures a systematic review and meta-analysis. JAMA Dermatology 2016;152(9):1008-14.

27. Phalen RN and Wong WK. Chemical resistance of disposable nitrile gloves exposed to stimulated movement. Journal of Occupational and Environmental Hygiene 2012;9(11):630-9.

28. Phalen RN and Wong WK. Tensile properties and integrity of cleanroom and low-modulus disposable nitrile gloves: a comparison of two dissimilar glove types. Annals of Occupational Hygiene 2011;56(4):450-7.

29. Phalen RN and Wong WK. Integrity of disposable nitrile exam gloves exposed to simulated movement. Journal of Occupational and Environmental Hygiene 2011;8(5):289-99.

30. Siew A. Ensuring Sterility of Parenteral Products. [online]. Iselin, NJ: Pharmaceutical Technology 2013. https://www.pharmtech.com/view/ensuring-sterility-parenteral-products [Accessed 26 November 2020].

31. Hall LB and Hartnett MJ. Measurement of bacterial contamination on surfaces in hospitals. Public Health Reports 1964;79(11):1021-4.

32. Dewhirst FE, Chen T, Izard J, et al. The human oral microbiome. Journal of Bacteriology 2010;192(19):5002-17.

33. Bodiwala GG and George TK. Surgical gloves during wound repair in the accident-and-emergency department. Lancet 1982;320(8289):912.

34. Hemsworth S, van Saene R and Pizer B. Does the number of exogenous infections increase in paediatric oncology patients when sterile surgical gloves are not worn for accessing central venous access devices? European Journal of Oncology Nursing 2007;11(5):442-7.

35. Osman OM and Jensen SL. Surgical gloves: current problems. World Journal of Surgery 1999;23:630-7.

36. Harno $\beta$ JC, Partecke LI, Hedecke CD, et al. Concentration of bacteria passing through puncture holes in surgical gloves. American Journal of Infection Control 2010;38(2):154-8. 
37. Partecke LI, Goerdt AM, Langner I, et al. Incidence of micro perforation for surgical gloves depends on duration of wear. Infection Control \& Hospital Epidemiology 2009;30(5):409-14.

38. Lloyd Jones M. 2.3 Non-sterile and sterile gloves: application and removal. British Journal of Healthcare Assistants 2014;8(2):64-7.

39. Johnson RL, Smith HM, Duncan CM, et al. Factors that influence the selection of sterile glove brand: a randomised controlled trial evaluating the performance and cost of gloves.

Canadian Journal of Anaesthesia 2013;60(7):700-8.

40. Carré MJ, Tan SK, Mylon PT, et al. Influence of medical gloves on fingerpad friction and feel, Wear 2017;376-377 (A):324-328.

41. Hughes KA, Theis JC and Brooks HJL. Bacterial contamination of unused, disposable non-sterile gloves on a hospital orthopaedic ward. Australasian Medical Journal 2013;6(6):331-8.

42. Zinner NL. How safe are your gloves? A study of protective barrier properties of gloves. AORN Journal 1994;59(4):876.

43. Muto CA, Sistrom MG, Strain BA, et al. Glove leakage rates as a function of latex content and brand. Archives of Surgery 2000;135(8):982-5.

44. Pittet $D$ and Boyce JM. Hand hygiene and patient care: pursuing the Semmelweis legacy. The Lancet Infectious Diseases 2001;(1):9-20.

45. Black RE, Dykes AC, Anderson KE, et al. Handwashing to prevent diarrhoea in day-care centers. American Journal of Epidemiology 1981;113(4):445-51.

46. A Chakrabarti, K Singh, A Narang, et al. Outbreak of pichia anomala infection in the paediatric service of a tertiary-care center in northern India. Journal of Clinical Microbiology 2001;39(5):1702-6.

47. Haverstick S. Patients' hand washing and reducing hospital-acquired infection. Critical Care Nurse 2017;37(3):e1-e8.

48. Dunn $\mathrm{H}$, Wilson $\mathrm{N}$ and Leonard $\mathrm{A}$. A programme to cut inappropriate use of non-sterile medical gloves. Nursing Times [online] 2019;115(9):18-20.

49. Fisher Scientific. Fisherbrand ${ }^{\mathrm{TM}}$ Comfort Nitrile Exam Gloves [online]. Fisher scientific UK, Loughborough, UK 2020; https://www.fishersci.co.uk/shop/products/fisherbrand-comfortnitrile-exam-gloves-18/15642367\#?keyword=true [Accessed 26 November 2020].

50. Fisher Scientific. Kimberly-Clark ${ }^{\mathrm{TM}}$ Professional Kimtech Pure ${ }^{\mathrm{TM}} \mathrm{G} 5$ Sterile Latex Gloves [online]. Fisher scientific UK, Loughborough, UK 2020;

https://www.fishersci.co.uk/shop/products/kimberly-clark-professional-kimtech-pure-g5-sterilelatex-gloves-8/p-140712\#?keyword=G5+GLOVES [Accessed 26 November 2020].

51. Blenkharn JI. Standards of clinical waste management in UK hospitals. Journal of Hospital Infection 2006;62:300-3.

52. Singh $\mathrm{S}$ and Prakash $\mathrm{V}$. Toxic environmental releases from medical waste incineration: A review. Environmental Monitoring and Assessment 2007;132:67-81.

53. Sawalem M, Selic E and Herbell JD. Hospital waste management in Libya: A case study. Waste Management 2009;1370-75. 